\title{
Dasatinib-induced Nonspecific Interstitial Pneumonia That Developed 7 Years after the Initiation of Dasatinib
}

\author{
Daisuke Takekoshi ${ }^{1}$, Yuma Matsui ${ }^{1}$, Takuya Akutsu ${ }^{1}$, Ayako Nishioka ${ }^{1}$, Ayu Kiritani ${ }^{1}$, \\ Keitaro Okuda ${ }^{1}$, Junko Watanabe ${ }^{1}$, Hanae Miyagawa ${ }^{1}$, Hirohumi Utsumi ${ }^{1}$, \\ Mitsuo Hashimoto ${ }^{1}$, Hiroshi Wakui ${ }^{1}$, Shunsuke Minagawa ${ }^{1}$, Hiromichi Hara ${ }^{1}$, \\ Takanori Numata ${ }^{1}$, Yuki Noda ${ }^{2}$, Rei Makishima ${ }^{3}$, Masahiro Ikegami $^{3}$, Yoshinori Kawabata ${ }^{4}$, \\ Jun Araya $^{1}$ and Kazuyoshi Kuwano ${ }^{1}$
}

\begin{abstract}
:
We report the case of a 56-year-old man with chronic myeloid leukemia (CML) who developed dasatinibinduced interstitial lung disease (ILD) 7 years after starting dasatinib, a BCR-ABL1 inhibitor. The patient presented with dyspnea. Chest imaging showed diffuse ground-glass opacities. A surgical lung biopsy showed cellular non-specific interstitial pneumonia (NSIP). Corticosteroid treatment ameliorated his condition. Bosutinib, another BCR-ABL1 inhibitor, was successfully re-instituted. The present case and relevant literature suggest that dasatinib-induced ILD can present as NSIP after an extended period, responds to corticosteroids, and is amenable to re-challenge at a lower-dose or with alternative BCR-ABL1 inhibitors.
\end{abstract}

Key words: chronic myeloid leukemia, dasatinib, interstitial lung disease, non-specific interstitial pneumonia

(Intern Med 59: 2297-2300, 2020)

(DOI: 10.2169/internalmedicine.4714-20)

\section{Introduction}

Dasatinib is a tyrosine kinase inhibitor that binds to the BCR-ABL1 oncoprotein that causes chronic myeloid leukemia (CML). In addition to blocking the BCR-ABL1 pathway, it inhibits several other signaling molecules, including SRC family kinases (e.g., SRC, LKC, YES, FYN), c-KIT, EPHA2 and PDGFR. Dasatinib is approved for CML with resistance or intolerance to imatinib, and for newly diagnosed CML. Pulmonary complications of dasatinib include pleural effusion, interstitial lung disease (ILD) and pulmonary arterial hypertension (1). Pleural effusion, which is the most common and best described of these, occurs in 12$30 \%$ of CML patients who take dasatinib (1-4). Pulmonary hypertension develops less often in $0.3-5 \%$ of patients (1-4). ILD is not so well described, but seems to be more common than is generally perceived $(2,4,5)$. We herein describe a case of dasatinib-induced ILD with cellular non-specific interstitial pneumonia (NSIP) histology, which developed 7 years after the initiation of dasatinib treatment, but which responded well to corticosteroids.

\section{Case Report}

A 56-year-old man with CML, which had been treated with dasatinib for 7 years, came to a local clinic complaining of dyspnea on exertion of 2 months' duration, which had significantly worsened during the prior week. As a chest $\mathrm{X}$-ray showed diffuse bilateral infiltration, he was referred to our hospital. Previously, he had started on dasatinib (100 mg daily) after treatment failure with imatinib and nilotinib. He achieved a major molecular response within 1 year after starting dasatinib and it had been maintained since that time. He took no other medications or dietary supplements, with the exception of loratadine, which he took as needed for fa-

\footnotetext{
${ }^{1}$ Division of Respiratory Medicine, Department of Internal Medicine, Jikei University School of Medicine, Japan, ${ }^{2}$ Division of Thoracic Surgery, Department of Surgery, Jikei University School of Medicine, Japan, ${ }^{3}$ Department of Pathology, Jikei University School of Medicine, Japan and ${ }^{4}$ Department of Pathology, Saitama Cardiovascular and Respiratory Center, Japan Received: February 22, 2020; Accepted: April 28, 2020; Advance Publication by J-STAGE: June 15, 2020 Correspondence to Dr. Daisuke Takekoshi, takekoshi@jikei.ac.jp
} 
A

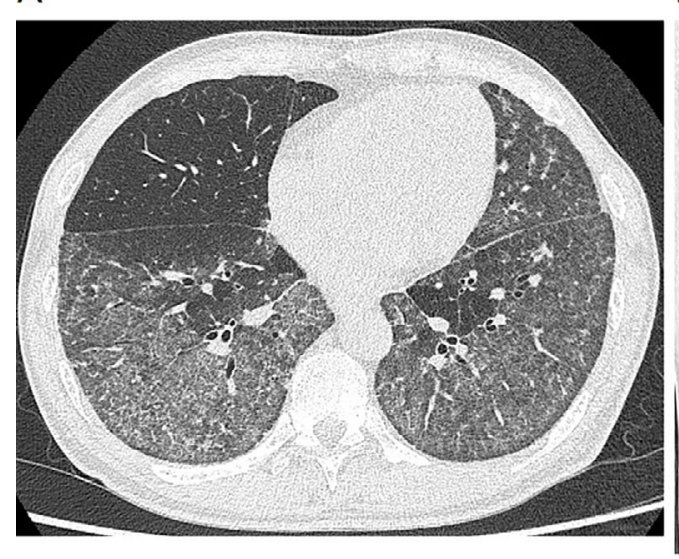

C

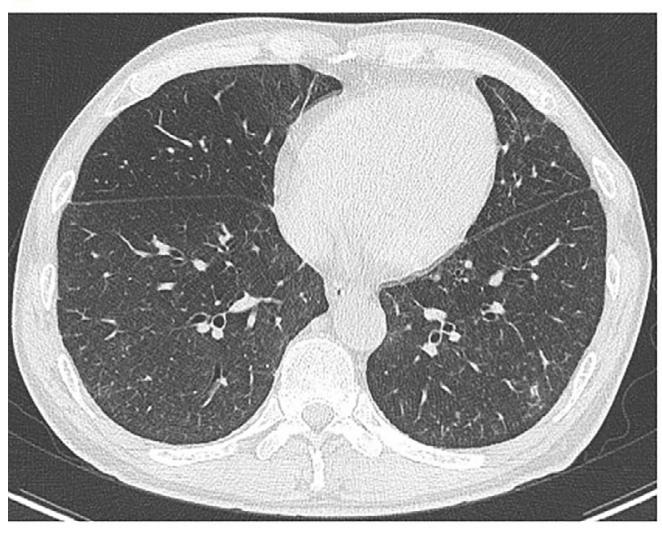

B

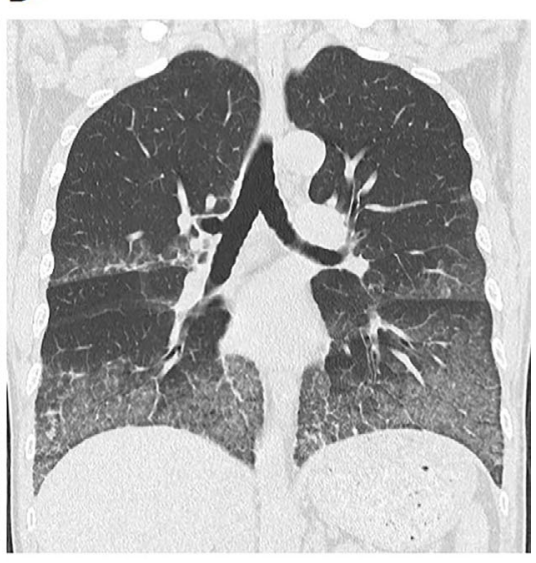

D

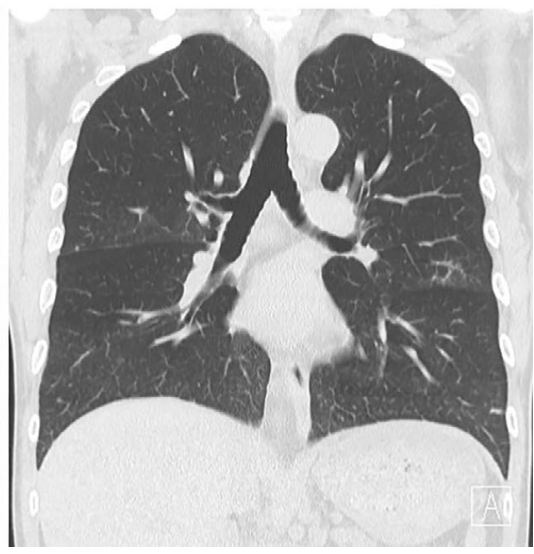

Figure 1. The Chest CT findings at presentation (A, B) and 2 weeks after corticosteroid treatment (C, D). At presentation, prevalent diffuse ground-glass opacities and mild interlobular septal thickening was observable predominantly in the lower lobes (A, B). Marked improvement was seen after 2 weeks of corticosteroid treatment $(C, D)$

cial rash for a few months until the 2 weeks prior to his presentation. His other past medical history included a benign parotid gland tumor that had been successfully resected. He was an ex-smoker with 20-pack-year history but had quit 18 years prior to his presentation.

On presentation, his vital signs were as follows: blood pressure, 136/66 mmHg; heart rate, $86 \mathrm{bpm}$; respiratory rate, 18 breaths/minute; and body temperature, $37.1^{\circ} \mathrm{C}$. His oxygen saturation was $90 \%$ at room air, which required $2 \mathrm{~L} / \mathrm{min}$ of supplemental oxygen through a nasal cannula with ambulation. Chest auscultation revealed fine crackles at his lower back. The rest of the examination was unremarkable.

Chest CT showed bilateral diffuse ground-glass opacities at the lower lobes and dependent areas, with mild thickening of the interlobular septa at the base of the lungs (Fig. 1A, B). Pleural effusion was not detected. His laboratory findings were as follows: WBC, 4,700 cells $/ \mu \mathrm{L}$ (neutrophils $69.6 \%$, lymphocytes $18.0 \%$, monocytes $6.4 \%$, eosinophils 4.9\%); CRP, $1.63 \mathrm{mg} / \mathrm{dL}$; LDH, $215 \mathrm{U} / \mathrm{L}$; KL-6, 3,326 $\mathrm{U} / \mathrm{mL}$; SP-D, $574 \mathrm{ng} / \mathrm{mL}$; BNP, $35.7 \mathrm{pg} / \mathrm{mL}$; and $\beta$-Dglycan $<6.0 \mathrm{pg} / \mathrm{mL}$. The serological panel for connective tissue diseases was all negative, including ANA, anti-ds DNA, anti-RNP, anti-SSA, anti-SCL-70, anti-CCP, MPO-ANCA,
PR3-ANCA, and anti-ARS. Anti-GM-CSF antibody was not detected. A cytomegalovirus antigen test was negative.

$\mathrm{He}$ was empirically treated with trimethoprimsulfamethoxazole and prednisolone for possible pneumocystis pneumonia. However, this treatment was stopped after 1 day, because PCR of bronchoalveolar lavage fluid (BALF) from the superior lingular segment (recovery rate: 69\%), taken at a bronchoscopic examination on the day of admission, was negative for $P$. jirovecii. The BALF analysis showed pleocytosis with predominant lymphocytes (total cell count: $1.88 \times 10^{6} / \mathrm{mL}$; macrophages: $10 \%$, lymphocytes: $88 \%$, neutrophils: $1 \%$, eosinophils: $1 \%$ ). The CD4/CD8 ratio was 2.12. We did not perform a transbronchial lung biopsy. Cultures, as well as bacterial, acid-fast bacillus and fungal staining were all negative.

Because these examinations were negative, dasatinibinduced ILD was suspected and dasatinib was discontinued. However, his condition did not improve after 2 weeks. At this point, we considered empirical treatment with corticosteroids, but secondary pulmonary alveolar proteinosis still remained as a possible differential diagnosis, on which corticosteroids may have a detrimental effect. Therefore, after a thorough discussion with the patient on its risks and 


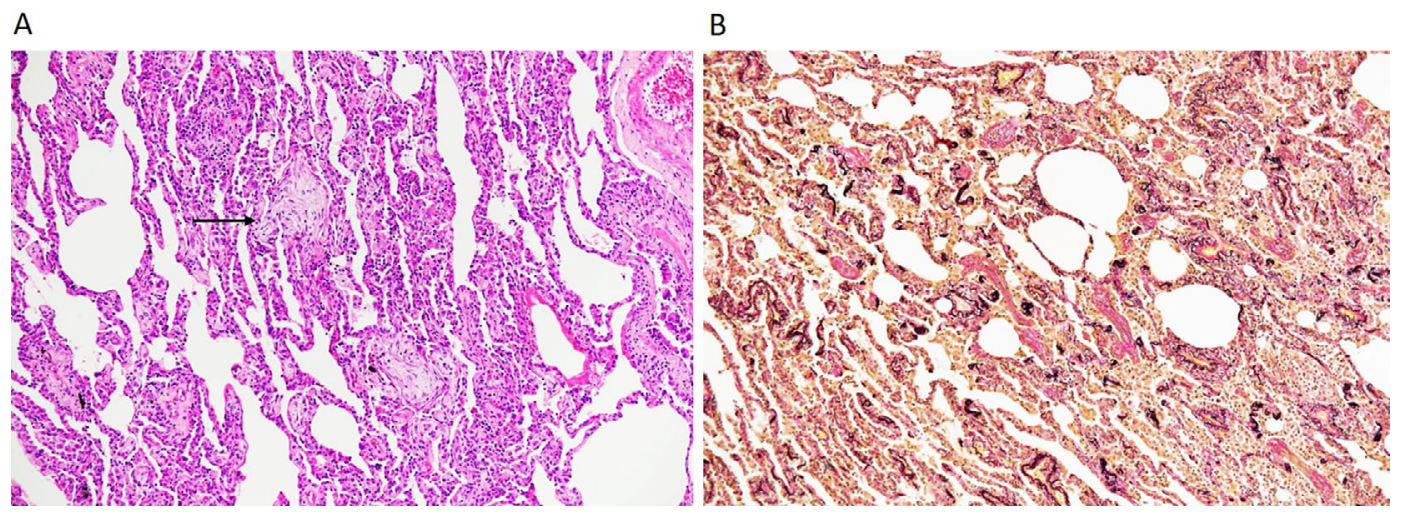

Figure 2. A surgical lung biopsy specimen shows diffuse lymphatic infiltration with some scattered organizing tissue formation (arrow; A, Hematoxylin and Eosin staining, $\times 100$ ) and an area with fibrotic changes $(B, E V G, \times 100)$. These findings are consistent with cellular NSIP. EVG: Elastica van Gieson stain, NSIP: non-specific interstitial pneumonia

benefits, we agreed to proceed to surgical lung biopsy of the upper and lower lobes. A pathological examination showed diffuse lymphocytic infiltration in the alveolar walls with some scattered organizing tissue formation and fibrotic changes, which are consistent with cellular nonspecific interstitial pneumonia (Fig. 2). We initiated a medium-dose corticosteroid treatment with prednisolone $(0.5 \mathrm{mg} / \mathrm{kg}$ daily). Within days, his respiratory condition improved; supplemental oxygen was tapered off within 4 days. CT images after 2 weeks of steroid treatment showed clear improvement (Fig. 1C, D). He was discharged to return home. The steroid dose was tapered to $10 \mathrm{mg}(0.15 \mathrm{mg} / \mathrm{kg})$ daily and bosutinib, another BCR-ABL inhibitor, was initiated. As of 8 months since the initiation of steroid therapy and 4 months after starting bosutinib, the steroid dose was further tapered to $2.5 \mathrm{mg}$ daily, without recurrence of ILD, even though bosutinib was intermittently withheld due to hepatotoxicity.

\section{Discussion}

There have been few reports on dasatinib-induced ILD $(2,5-8)$. However, they appear to show some common features: (a) occurrence long after the use of dasatinib (length of time between starting dasatinib and the onset of ILD varies widely), (b) lymphocyte-predominant inflammation, (c) good response to steroid treatment, and (d) tolerability to re-challenge.

The time to the onset ranges from only a few days to several years after the start of dasatinib (6). ILD developed in 32 of 897 patients $(3.6 \%)$ in post-marketing surveillance of all patients who received dasatinib in Japan from March 2009 to December 2009 (4). This surveillance found that, although most ILD occurred during the first 90 days, ILD continued to develop sporadically over about 700 days during the 4-year observation period. An analysis of the timeto-onset of ILD after starting dasatinib, using the national database of spontaneously reported drug adverse reactions showed that the median time of onset for dasatinib-induced ILD was 31.5 days (range: 3-1,407 days) (9). This analysis also calculated Weibull distribution parameters and found that the probability of dasatinib-induced ILD tended to decrease as time passed (i.e., the Weibull distribution parameter was $<1)$. Therefore, overall, dasatinib-induced ILD tends to occur in the first few months, similarly to other druginduced adverse events; however, ILD does develop a long time after the initiation of treatment-as was observed in our case-in a significant number of cases.

BALF analyses mostly showed lymphocytic predominance $(2,5,6,8)$. The CD4/CD8 ratio in our case was 2.12 ; however, the $\mathrm{CD} 4 / \mathrm{CD} 8$ ratio was rarely mentioned in previous reports of dasatinib-induced ILD. Among 14 reported cases, only 4 mentioned the $\mathrm{CD} 4 / \mathrm{CD} 8$ ratio $(2,5-8)$. The reported $\mathrm{CD} 4 / \mathrm{CD} 8$ ratios were $1.0,2.9,4.8$ in one case series (2), and 2.1 in one case report (8). Thus, it is difficult to point out a clear association between the CD4/CD8 ratio and dasatinib-induced ILD. Although lung biopsy results have rarely been reported, the few reported cases (per transbronchial lung biopsy) demonstrated no abnormality or organizing pneumonia patterns $(2,8)$. Thus, we believe this is the first case of dasatinib-induced ILD to show cellular NSIP based on a surgical lung biopsy. Interestingly, a detailed pathological examination elucidated the focal existence of an organizing pneumonia pattern in our case. Unsurprisingly, the same drug induces different ILD pathological patterns. Extensive pathological examination may elucidate the coexistence of different ILD patterns, especially with sufficiently large samples from surgical lung biopsies. Accordingly, previously reported organizing pneumonia patterns in transbronchial lung biopsy samples may not reflect the overall pathological processes.

With respect to the clinical outcome, most reported patients with dasatinib-induced ILD, including ours, responded well to treatment. In many cases, they improved only after drug interruption. When merely interrupting dasatinib was not effective, they usually improved with corticosteroid treatment $(2,5-8)$. No mortality has been reported thus far (5). Furthermore, the successful re-administration of dasatinib, at lower doses or with corticosteroids, has been 
reported $(2,5,8)$. In one case, the ILD resolved with corticosteroid treatment without the discontinuation of dasatinib (2). Favorable experiences with re-administration indicate that it is a plausible treatment option, especially considering the critical role that ABL-TKIs play in treating CML. Cross-reactivity against other ABL-TKIs (such as nilotinib and bosutinib) is unclear. However, successful switching of ABL-TKIs has been reported in several cases without recurrence of ILD (10).

Together with the lymphocyte-predominant inflammation in BALF and surgical lung biopsy samples, a good clinical response to corticosteroids indicates that an immunologic mechanism underlies the pathogenesis. In addition, as lowering the dasatinib dose can decrease these side effects $(1,11)$, dasatinib appears to disturb the immune system in a dose-dependent manner, making a hypersensitivityallergic type reaction less likely. The exact mechanisms of ABL-TKI-induced ILDs are unclear. Pulmonary complications could be a class effect of ABL-inhibition, because other ABL-TKIs have also been implicated in drug-induced ILDs (1). However, pulmonary complications seems to be more common in dasatinib (mainly in the form of pleural effusion) (11). The differences in ABL-TKI toxicity profiles may partly reflect differences in the inhibition of non-ABLkinases (e.g., FGFR1, FLT3, KIT, PDGFR, or SRC family) $(1,12,13)$. Some authors have pointed out that the SRC-family kinases, LCK and LYN, which are targets of dasatinib, are expressed in B- and T lymphocytes and may affect the pathogenesis of ILD (2). PDGFR, which is also a target of dasatinib, is implicated in acute lung injury $(14,15)$. In comparison to other ABL-TKIs, dasatinib can modulate the immune response by efficiently expanding the memory $\mathrm{T}$ cell population (16). Leukocytosis/lymphocytosis is commonly seen in patients treated with dasatinib (17). Even though the enhanced immune reaction may improve leukemia control, it has been reported that dasatinib-induced lymphocytosis may be associated with increased side effects, such as pleural effusion (17). Based on our pathological findings in the lung biopsy sample, it is plausible that an immunomodulatory effect may be involved in the mechanisms of dasatinib-induced ILD, even in the absence of peripheral lymphocytosis. However, the exact mechanism of dasatinib-induced ILD is unclear and further studies are needed.

In conclusion, we present a case of dasatinib-induced ILD that developed long after starting dasatinib in a patient with well-controlled CML. Surgical lung biopsy showed cellular NSIP histology with rich lymphocytic infiltration. Consistent with previous reports, it responded well to corticosteroids, and an alternative ABL-TKI was successfully initiated with low-dose corticosteroid treatment. Although it is rare, internists should be aware of dasatinib-induced ILD, even after long-term treatment for CML.

The authors state that they have no Conflict of Interest (COI).

\section{Acknowledgement}

We thank Marla Brunker for editing a draft of this manuscript.

\section{References}

1. Caldemeyer L, Dugan M, Edwards J, Akard L. Long-term side effects of tyrosine kinase inhibitors in chronic myeloid leukemia. Curr Hematol Malig Rep 11: 71-79, 2016.

2. Bergeron A, Rea D, Levy V, et al. Lung abnormalities after dasatinib treatment for chronic myeloid leukemia: a case series. Am J Respir Crit Care Med 176: 814-818, 2007.

3. Quintas-Cardama A, Kantarjian H, O'Brien S, et al. Pleural effusion in patients with chronic myelogenous leukemia treated with dasatinib after imatinib failure. J Clin Oncol 25: 3908-3914, 2007.

4. Bristol-Myers Squibb Healthcare i. post-marketing surveillance reports on Sprycel [Internet]. [cited 2019 Sep 9]. Available from: htt p://file.bmshealthcare.jp/sprycel/pdf/file/sp_kaiseki.pdf (in Japanese).

5. Ito $\mathrm{Y}$, Tanigawa $\mathrm{M}$, Iwamoto $\mathrm{K}$, et al. Interstitial pneumonitis associated with dasatinib: two case reports and literature review. Respir Investig 57: 506-509, 2019.

6. Jasielec JK, Larson RA. Dasatinib-related pulmonary toxicity mimicking an atypical infection. J Clin Oncol 34: e46-e48, 2016.

7. Radaelli F, Bramanti S, Fantini NN, Fabio G, Greco I, Lambertenghi-Deliliers G. Dasatinib-related alveolar pneumonia responsive to corticosteroids. Leuk Lymphoma 47: 1180-1181, 2006.

8. Sato M, Watanabe S, Aoki N, et al. [A case of drug-induced organizing pneumonia caused by dasatinib]. Gan To Kagaku Ryoho (Cancer Chemother) 45: 851-854, 2018 (in Japanese, Abstract in English).

9. Komada F. [Analysis of time-to-onset of interstitial lung disease after the administration of small molecule molecularly-targeted drugs]. Yakugaku Zasshi (J Pharmacent Soc Japan) 138: 229-235, 2018 (in Japanese, Abstract in English).

10. Delomas T, Darne $C$, Besson C. Lack of recurrence of imatinibinduced interstitial lung disease with nilotinib. Leuk Lymphoma 53: 332-333, 2012.

11. Rea D. Management of adverse events associated with tyrosine kinase inhibitors in chronic myeloid leukemia. Ann Hematol 94 Suppl 2: S149-S158, 2015.

12. Deininger MW, Manley P. What do kinase inhibition profiles tell us about tyrosine kinase inhibitors used for the treatment of CML? Leuk Res 36: 253-261, 2012.

13. Uitdehaag JC, de Roos JA, van Doornmalen AM, et al. Comparison of the cancer gene targeting and biochemical selectivities of all targeted kinase inhibitors approved for clinical use. PLoS One 9: e92146, 2014

14. Snyder LS, Hertz MI, Peterson MS, et al. Acute lung injury. Pathogenesis of intraalveolar fibrosis. J Clin Invest 88: 663-673, 1991.

15. Walsh J, Absher M, Kelley J. Variable expression of plateletderived growth factor family proteins in acute lung injury. Am J Respir Cell Mol Biol 9: 637-644, 1993.

16. Kreutzman A, Ilander M, Porkka K, Vakkila J, Mustjoki S. Dasatinib promotes Th1-type responses in granzyme B expressing T-cells. Oncoimmunology 3: e28925, 2014.

17. Schiffer CA, Cortes JE, Hochhaus A, et al. Lymphocytosis after treatment with dasatinib in chronic myeloid leukemia: Effects on response and toxicity. Cancer 122: 1398-1407, 2016.

The Internal Medicine is an Open Access journal distributed under the Creative Commons Attribution-NonCommercial-NoDerivatives 4.0 International License. To view the details of this license, please visit (https://creativecommons.org/licenses/ by-nc-nd/4.0/).

(C) 2020 The Japanese Society of Internal Medicine

Intern Med 59: 2297-2300, 2020 